EOMmun Communication et organisation

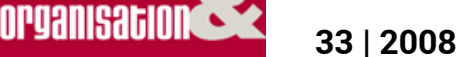

Conduire le changement organisationnel ?

\title{
La nouvelle équipe de recherche Cosmos de l'université de Bourgogne
}

Pour une dialogique Organisations/Savoirs

Daniel Raichvarg et Jacques Bonnet

\section{OpenEdition}

\section{Journals}

Édition électronique

URL : http://journals.openedition.org/communicationorganisation/536

DOI : 10.4000/communicationorganisation.536

ISSN : $1775-3546$

Éditeur

Presses universitaires de Bordeaux

Édition imprimée

Date de publication : 1 juin 2008

Pagination : 139-142

ISBN : 978-2-86781-506-5

ISSN : 1168-5549

\section{Référence électronique}

Daniel Raichvarg et Jacques Bonnet, «La nouvelle équipe de recherche Cosmos de l'université de Bourgogne », Communication et organisation [En ligne], 33 | 2008, mis en ligne le 01 juin 2008, consulté le 21 septembre 2020. URL : http://journals.openedition.org/communicationorganisation/536 ; DOI : https://doi.org/10.4000/communicationorganisation.536 


\title{
La nouvelle équipe de recherche Cosmos de l'université de Bourgogne Pour une dialogique Organisations/Savoirs
}

\author{
Daniel Raichvarg, Jacques Bonnet
}

daniel.raichvarg@u-bourgogne.fr

j.bonnet@enesad.fr

L'équipe de recherche COSMOS (COmmunication, Savoirs, Médiations et OrganisationS, dir. Jacques Bonnet/Enesad), est l'une des trois composantes de l'Equipe d'Accueil CIMEOS (EA n¹77, dir. Daniel Raichvarg) de l'Université de Bourgogne. Elle a pour objectif de développer une approche informationnelle et communicationnelle des importants changements culturels et socioéconomiques qui affectent les organisations, dans leurs identités, leurs messages et leurs activités. Ces changements sont considérés comme des processus et des productions de signification individuelle et collective, en tant que témoignages du rapport que les individus, les groupes et les sociétés construisent et entretiennent avec une réalité mouvante et complexe. C'est pourquoi les concepts de représentation, de valeur, d'identité, de culture(s), de logiques d'acteurs, de formes et de symboles, prennent, dans la perspective scientifique de Cosmos, une place importante pour traiter des rapports aux savoirs, aux usages et aux pratiques sociales et/ou professionnelles, aux projets, aux changements, aux messages et aux images dans les contextes formels ou informels où se construisent et se déroulent les activités humaines. Cosmos s'inscrit ainsi dans le champ des Sciences de l'information et de la communication dans une perspective de recherche qui place le problème de l'élaboration et du partage du sens au cœur des analyses et des réflexions, notamment à travers des questionnements liés :

- aux nouveaux rapports individuels et collectifs envers les savoirs (savoirs populaires, savoirs d'action, savoirs savants), le travail et les organisations (administrations, collectivités territoriales, entreprises, associations), aux nouvelles logiques de raisonnement et d'action ainsi qu'aux nouveaux comportements des acteurs dans et autour de ces contextes plus ou moins formels,

- à la forte rotation des classes d'âge d'actifs et à ses effets en 


\section{Etat de la recherche}

matière de chocs de cultures et de pratiques et, plus généralement, en termes de lien social et d'interculturalité,

- aux politiques, aux pratiques et aux messages des institutions publiques et des acteurs socio-économiques privés (diffusés dans les médias ou par d'autres vecteurs), par exemple en matière de développement durable et, plus globalement, de viabilité et de fiabilité économiques et sociales face à des questions technoscientifiques relatives à l'éducation à la santé, à l'environnement.

Les principaux axes de recherche de Cosmos concernent :

- l'évolution des modes de construction, de diffusion et de circulation des savoirs dans les organisations et les médias, les pratiques organisationnelles et communicationnelles dites «apprenantes", notamment en matière de médiations, de discours, de transparence et de communication interne, d'émergence de nouveaux vecteurs et de nouvelles formes de savoirs impulsées par les acteurs à travers les supports numériques et la communication électronique, de systèmes et réseaux d'information et d'échanges d'expériences,

- les processus, les pratiques et les parcours de professionnalisation des ingénieurs, cadres et dirigeants des organisations, la place réservée à l'élaboration du sens dans les processus et les dispositifs de développement professionnel et de socialisation des acteurs,

- les processus d'impulsion et de conduite du changement et de l'innovation dans les organisations, leurs formes de diffusion dans les médias et d'autres supports, leur(s) signification(s) pour les acteurs concernés, en termes d'identité, de coopération et d'intercompréhension,

- les pratiques de «nouvelle gouvernance » au regard de l'évolution de la professionnalité et des activités des cadres et des dirigeants confrontés aux exigences et à l'exercice complexe d'une fonction d'interface fonctionnelle et symbolique, sa contribution à l'évolution de l'image sociale de la responsabilité et de la citoyenneté, y compris face aux problèmes environnementaux.

Basé à Dijon, à l'Université de Bourgogne, Cosmos réunit une dizaine de chercheurs et chercheurs associés. Un séminaire constitutif a eu lieu en décembre 2007 en présence de Gino Gramaccia, Président de la Sfsic. Les deux journées de ce séminaire ont été consacrées à la 
présentation et à la mise en débat d'axes de recherche permettant de contribuer à la formalisation de la politique scientifique de l'équipe. Ces travaux se sont appuyés sur les avis et les apports croisés d'universitaires, de professionnels, de représentants de différents organismes et institutions, réunis au titre de leur expertise scientifique et/ou technique. La mise en relation de ces différentes sources et formes d'expertise a permis d'étayer, tant sur le fond que sur la forme, l'ambition qui préside au projet de l'équipe Cosmos : la mise en dialogue de la recherche et des réalités culturelles et socioprofessionnelles relatives aux savoirs et aux organisations.

A la suite de ce séminaire, un colloque intitulé «Société, organisations et nouveaux modèles de savoirs - Recherches et pratiques communicationnelles » est prévu les 27 et 28 novembre 2008 à Dijon. Le champ de questionnement posé est le suivant : des Encyclopédies du Siècle des Lumières à Wikipédia, des ouvriersgraveurs du $\mathrm{XIX}^{\mathrm{e}}$ siècle aux webmaîtres, des associations philanthropiques et d'éducation populaires aux collectifs antiSida et autres ONGs, de la presse populaire à $X$-files, des «house organs » à l'Intranet, de l'image de marque à la communication sur le développement durable, du commandement vertical et cloisonné des activités à la quête de gouvernance... Continuités des modes de construction et de circulation des savoirs dans les organisations et les médias ? Changements? Ruptures?

Ce colloque propose de rassembler des contributions de recherche autour du développement de pratiques organisationnelles et communicationnelles dites apprenantes, de l'émergence de nouveaux vecteurs et de nouvelles formes de savoirs impulsés par les acteurs au travers de systèmes et de réseaux d'informations et d'échanges de pratiques et d'expériences.

Trois axes ont été retenus pour ce colloque, dans une logique communicationnelle, interculturelle et diachronique.

Axe 1 - Les acteurs des savoirs : leurs cultures, leurs logiques, leurs pratiques, leurs représentations du -et des- savoir(s), les formes symboliques de leur engagement dans l'émergence et la diffusion des savoirs.

Axe 2 - Les processus et les dispositifs d'émergence, de prise en compte et d'accueil, d'essaimage et d'échange, relatifs aux savoirs dans les organisations (entreprises, collectivités territoriales, administrations, associations, communautés scientifiques). 


\section{Etat de la recherche}

Axe 3 - Les formes du quotidien en tant que vecteurs de savoirs et de pratiques communicationnelles: journaux et magazines populaires, catalogues, almanachs, séries télévisées et fictions de sciences.

Les collègues intéressés peuvent faire parvenir un résumé de 1500 signes avant le 15 juin 2008 par courrier postal :

Université de Bourgogne

D. Raichvarg, 36 rue Chabot-Charny 21000 Dijon

ou électronique à :

j.bonnet@enesad.fr ET Daniel.Raichvarg@u-bourgogne.fr

Le programme du colloque sera adressé le 30 juin.

Les communicants bénéficieront d'un temps d'expression de vingt minutes. Les frais d'inscription au colloque, comprenant les actes, les pauses café et les déjeuners, sont de 100 euros par participant pour 2 jours (50 euros pour les doctorants, gratuit pour les doctorants de l'Université de Bourgogne). 